陈思 ${ }^{1}$, 史继云 ${ }^{2}$, 王凡 ${ }^{2,3 *}$

1. 佛山原子医疗设备有限公司, 佛山 528200 ;

2. 中国科学院生物物理研究所蛋白质与多肽药物实验室, 北京 100101 ;

3. 北京大学医学同位素研究中心, 北京 100191

* 联系人: E-mail: wangfan@bjmu.edu.cn

收稿日期：2020-07-22; 接受日期：2020-09-14; 网络版发表日期：2020-10-09

摘要核医学影像在疾病的诊断、分期、预后、疗效评价以及指导个体化治疗方面发挥着不可替代的作用, 已 在临床广泛应用。核医学影像的发展取决于显像剂和显像设备的不断进步。我国核医学已走过60多年的发展历 程, 完成了药物和设备的从进口到国产替代的转变, 如今进入自主创新的发展阶段. 本文叙述了放射性药物和核 医学影像仪器的发展和现状, 并对我国核医学的发展进行了展望.

关键词核医学影像, 放射性药物, SPECT, PET, 诊断, 治疗

作为现代医学技术之一, 核医学影像已得到广泛 的应用, 特别是在当今精准医学时代, 核医学分子影 像以及多模态分子影像已成为核医学影像的主流, 在 疾病的诊断、分期、预后、疗效评价以及指导个体化 治疗方面发挥着不可替代的作用. 我国核医学已走过 60 多年的发展历程, 完成了药物和设备的从进口到国 产替代的转变, 如今进入自主创新的发展阶段.

\section{1 核医学影像技术的发展历程}

核医学影像(nuclear medicine imaging)也称核素成 像(radionuclide imaging), 是根据放射性核素示踪原理, 利用放射性核素或其标记化合物在体内分布的特殊规 律, 从体外获得脏器和组织功能结构影像的一种技术.
用于脏器、组织或病变显像的放射性核素或其标记化 合物称为显像剂(imaging agent). 核医学影像的发展主 要取决于显像剂和显像设备的不断进步. 从 20 世纪 50 年代至今, 核医学影像技术的发展历程大致可以分为 三个阶段：1950 1990，诞生和快速发展；1990 2005, 由功能影像向融合影像突破与转型; 2005至今, 分子 影像.

核医学显像设备主要包括两种, 单光子发射型计 算机断层仪(single photon emission computed tomography, SPECT)与正电子发射型计算机断层仪 (positron emission computed tomography, PET). 早期的单光子 成像设备可以追溯到20世纪50年代. 1948年, Hofstadter开发了用于 $\gamma$ 闪炼测量的碘化钠晶体. 1951年, 美国 加州大学Cassen成功研制第一台闪㧎扫描机, 并获得

引用格式: 陈思, 史继云, 王凡. 加强我国核医学分子影像技术的自主创新发展. 中国科学: 生命科学, 2020, 50: 1192-1201

Chen S, Shi J Y, Wang F. Strengthening the independent innovation and development of nuclear medicine molecular imaging in China (in Chinese). Sci Sin Vitae, 2020, 50: 1192-1201, doi: 10.1360/SSV-2020-0168 
了第一幅人的甲状腺扫描图, 莫定了核医学影像的基 础. 1957年, Anger研制出第一台 $\gamma$ 照相机, 与核素扫描 仪相比, $\gamma$ 相机的分辨率明显提高, 并使得核医学从静 态显像进入动态显像成为可能, 是核医学显像技术 的一次飞跃性发展. 1976年, Keyes和Jaszezak分别成 功研制第一台通用型 SPECT 和第一台头部专用型 SPECT, 实现了核素断层显像, 与传统放射性核素扫描 仪和 $\gamma$ 相机相比, SPECT除了增加断层显像功能外, 探 测器和准直器技术的不断进步也使其在灵敏度、分辨 率和均匀性等重要性能指标方面均有很大提高. 1991 年, 美国加州大学旧金山分校的Hasegawa和Lang等人 最早探索将SPECT与CT组合成双功能医学成像系统, 在此基础上, 将两种成熟的临床专用设备组装到同一 台机器上, 即将一台 GE $600 \mathrm{XR} / \mathrm{T}$ 型SPECT仪和一台 GE 9800型CT串联在一起, 历史上首次实现了双模态 融合成像. 1996年, Blankespoor等人首先报道这一设备 在心肌灌注显像中的应用. 1998年, GE公司将基于这 一设计的Hawkeye系列SPECT/CT推向市场, 其中的 CT模块与SPECT共享同一机架, 通过慢速旋转扫描获 得的CT图像用于SPECT衰减校正和解剖定位, 获得巨 大的成功. 2004年, 西门子公司在第51届美国核医学年 会上提出了一种新的融合影像技术概念, 首次将 SPECT 的功能影像与多层诊断CT的丰富解剖细节进 行了充分的结合, 推出了新型True Point 8482 SPECT/ CT. 至此, SPECT/CT融合成像技术基本定型, 并逐步 在临床普及.

与单光子成像设备类似, 早期的正电子成像设备 也可以追溯到20世纪50年代 ${ }^{[1]}$. 1950年, Brownell等人 研制了双单通道探测器正电子符合探测系统, 并通过 二维机械运动实现头部扫描成像功能. 在 60 年代, Brownell研究组进一步开发了双多通道探测器的正电 子符合系统, 并在 70 年代初升级为双二维面阵平板探 测器系统PC-I. PC-I首先通过摆动实现断层成像, 随着 滤波反投影算法的开发和应用，进一步实现了计算断 层成像. 1975年, Ter-Pogossian等人成功研制出第一台 基于环形探测器的正电子符合成像系统, 确定了 PET 的主流技术路线, 并沿用至今. 20 世纪70年代, 电子计 算机广泛应用于核医学领域, 核医学成像由定性分析 进入定量分析阶段, 由平面影像进入断层影像阶段, 促进了SPECT和PET显像的发展. 1974年首台商业 PET诞生, 1992年PET用于临床全身显像. 随着技术的
发展，PET的分辨率、灵敏度以及成像效率不断得到 提高. 1995年, Townsend等人研制出集PET与CT于一 体的PET/CT. 随着2000年PET/CT在北美放射年会上 被展示, 2001年之后PET/CT广泛应用于临床, 以 $\mathrm{PET}$ 为代表的分子功能影像与以 $\mathrm{CT}$ 为代表的形态学影像 实现了完美的结合, 成为当今多模态分子影像的代表.

SPECT/CT和PET/CT多模态融合成像设备在临床 上的应用极大地推动了核医学显像技术的发展和普 及. 2011年, 西门子在全球首先推出PET/MRI一体机, 虽然在技术细节和临床应用方法等方面仍有进一步完 善和提升的空间, 临床价值也有待进一步挖掘, 但其作 为全球最高端医学影像设备之一, 对于核医学影像临 床地位的提升有重要意义.

核医学影像被应用于脑、心、肾、肺、甲状腺及 肿瘤等多种器官和组织的显像中, 评价其血流灌注、 代谢状况、受体表达水平等多种指标, 在疾病诊断、 病程分期和疗效监测上发挥着重要作用. 与之相对应, 不同的放射性药物在临床得到应用. ${ }^{99 \mathrm{~m}} \mathrm{Tc}-\mathrm{MIBI}$ (锝 $\left[{ }^{99 \mathrm{~m}} \mathrm{Tc}\right]$ 司它比注射液)心肌灌注显像是对冠心病心肌 缺血的早期诊断与危险度分级的最常用的基本手段 ${ }^{[2]}$; ${ }^{99 \mathrm{~m}} \mathrm{Tc}-\mathrm{MDP}$ (锝 $\left.{ }^{99 \mathrm{~m}} \mathrm{Tc}\right]$ 亚甲基二膦酸盐)骨显像是目前 诊断骨转移性肿瘤最敏感的方法 ${ }^{[3]} ;{ }^{99 m} \mathrm{Tc}-\mathrm{DTPA}$ (锝 $\left[{ }^{99 m} \mathrm{Tc}\right]$ 二乙三胺五乙酸注射液) 和 ${ }^{99 m} \mathrm{Tc}-\mathrm{MAG} 3$ (锝 $\left[{ }^{99 \mathrm{~m}} \mathrm{Tc}\right]$ 颈乙甘肽注射液)用于评价肾功能 ${ }^{[4]} ;{ }^{99 \mathrm{~m}} \mathrm{Tc}-\mathrm{PYP}$ (锝 $\left[{ }^{99 \mathrm{~m}} \mathrm{Tc}\right]$-焦磷酸盐) 用于急性心肌梗死灶显像 ${ }^{[5]}$; ${ }^{99 \mathrm{~m}} \mathrm{Tc}-\mathrm{MAA}\left(\right.$ 锝 $\left.{ }^{99 \mathrm{~m}} \mathrm{Tc}\right]$-大颗粒聚合人血清白蛋白)用于 肺通气与灌注显像诊断肺栓塞 ${ }^{[6]} ; \mathrm{Na}^{99 \mathrm{~m}} \mathrm{TcO}_{4}$ 用于甲状 旁腺显像诊断甲状旁腺功能六进 ${ }^{[7]} ;{ }^{99 \mathrm{~m}} \mathrm{Tc}$-硫胶体用于 全身骨髓显像判断全身骨髓活性 ${ }^{[8]} ;{ }^{99} \mathrm{~m}$ Tc-HMPAO(锝 $\left[{ }^{99 \mathrm{~m}} \mathrm{Tc}\right]$ 依莎美肜注射液) 和 ${ }^{99 \mathrm{~m}} \mathrm{Tc}-\mathrm{ECD}\left(\mathrm{c}_{\mathrm{g}}\left[{ }^{99 \mathrm{~m}} \mathrm{Tc}\right]\right.$ 双半胱 乙酯注射液)用于脑显像评价脑功能 ${ }^{[9]}$. 这些SPECT显 像剂都在临床上常规使用, 数十年经久不衰. 20 世纪 70年代，美国布鲁克海文国家实验室(Brookhaven National Laboratory)的Ido等人 ${ }^{[10]}$ 首先完成了 ${ }^{18}$ F-FDG ( ${ }^{18}$ 氟-脱氧葡萄糖)的合成. 1972年, Kuhl博士作为主要 成员应用三维显示法和 ${ }^{18} \mathrm{~F}-\mathrm{FDG}$ 测定了脑局部葡萄糖 的利用率, 成为利用PET进行葡萄糖代谢显像的基础.

${ }^{18} \mathrm{~F}-\mathrm{FDG}$ 作为应用最广泛的PET显像剂, 绝大部分应用 于肿瘤诊断、分期和疗效评估，仅少部分用于心肌活 性和脑功能评价 ${ }^{[11]}$. 此外, 反映肿瘤细胞增殖的显像 
剂 ${ }^{18} \mathrm{~F}-\mathrm{FLT}\left({ }^{18} \mathrm{~F}-3^{\prime} \text { - 脱氧胸腺嘧啶核甘 }\right)^{[12]}$, 反映组织乏 氧的显像剂 ${ }^{18} \mathrm{~F}-\mathrm{FMISO}\left({ }^{18} \mathrm{~F} \text {-氟硝基咪唑 }\right)^{[13]}$, 以 及 ${ }^{18} \mathrm{~F} 、{ }^{11} \mathrm{C}$ 标记的胆碱、乙酸氨基酸代谢显像剂也都 在临床上得到应用 ${ }^{[14]}$.

\section{2 核医学影像进入分子影像发展阶段}

随着分子生物学等相关领域技术的发展，核医学 影像也进入到分子影像发展阶段. 1999年，美国哈佛 大学Weissleder教授 ${ }^{[15]}$ 提出分子影像学(molecular imaging, MI)的概念，即应用影像学的方法对活体状态下 (in vivo) 的生物过程进行细胞和分子水平的定性和定 量研究, 从而在活体状态下直接观察疾病起因、发 生、发展等一系列的病理生理变化和特征，以及迅速 评估药物对疾病的疗效并高效指导新药研发. 分子影 像在分子生物学与临床医学之间架起了桥梁，被美国 医学会评为未来最具有发展潜力的十个医学科学前沿 领域之一, 是 21 世纪的影像学. 在包括光学显像、磁共 振成像(magnetic resonance imaging, MRI)、超声成像 的各种分子影像模态当中，核医学分子影像率先进入 临床应用. 在 21 世纪的第一个 10 年，核医学积极拥抱 分子影像概念, 其中 ${ }^{18}$ F-FDG PET/CT毫无疑问是核医 学的旗帜, 同时是分子影像学概念的重要源头和实践 基础 ${ }^{[16]}$. 2010年以后，世界各国纷纷将核医学学会更 名为核医学与分子影像学会，除了自身学科特点与分 子影像十分契合以外, 还有外部环境的原因. 从医学 影像技术的竞争层面来说，CT和MRI在解剖成像的分 辨率、对比度以及灵活性方面持续提升，并向功能影 像领域拓展(如灌注成像、动态成像等), 不断冲击核 医学临床应用的传统领域——脑、肺和心肌显像等. 同期核医学影像设备技术虽然也在不断创新，但在进 步速度、性价比以及易普及性方面，与解剖成像相比 仍处于下风. 因此，核医学影像技术迫切需要回归和 强化自己的核心竞争力一一借助于特异性放射性药物 实现从细胞和分子水平对人体生化过程的高灵敏度观 测, 从而赢得在新世纪的发展机遇. 从大环境来说, 这 个机遇是确实存在的. 21 世纪以来, 随着人口增长和平 均寿命的提升，恶性肿瘤等重大慢性病的死亡率有所 降低，但发病率仍居高不下，造成医疗费用和社会资 源的巨大负担. 如何在满足公众健康需求的前提下控 制成本，就成为当今世界主要经济体医学技术发展迫
切要解决的核心问题，以早诊早治和个性化治疗为主 要特征的精准医学概念应运而生. 在精准医学体系中, 无论是疾病早期诊断、指导治疗还是提升新药的研发 效率, 都为核医学分子影像提供了用武之地.

随着核医学分子影像的发展，核医学设备和核医 学药物也得到快速发展. 在PET设备方面, 当前两个代 表性的新技术分别是TOF(time of flight)技术和PET/ MRI一体机技术. TOF 是通过测定正电子湮灭时发出 的一对光子到达探测器的飞行时间差而直接计算出湮 灭发生的具体位置. 探测器时间分辨率越高, 通过TOF 信息计算正电子湮灭发生位置的不确定性就越小，其 包含的信息量越大，最终重建PET图像的信噪比提升 就越明显. 目前，业界最新的TOF PET设备是西门子 公司的Biograph Vision，可以达到 214 ps的TOF时间分 辨率, 相对于早期(2005年左右)TOF PET系统的500 ps 以上的TOF时间分辨率有了显著的提升 (https://www. siemens-healthineers.com $)^{[17]}$. 这一技术的提升得益于 在PET探测器模块中采用硅光电倍增管(silicon photomultiplier, SiPM)取代传统真空光电倍增管. SiPM一个 模块是由许多微像素的单元(cell)组成的阵列, 每个微 像素单元都是盖革模式的单光子计数器, 像素单元阵 列联合可以输出正比于入射光子数目的数字或模拟电 流信号, 从而实现光电转换. 与传统真空光电倍增管相 比，当前的SiPM技术具有工作电压低、体积小、探测 效率高、时间响应快、磁场兼容等特点. 由于 SiPM是 半导体技术, 未来进一步优化和降低成本仍有较大空 间. 基于半导体的光电转换器件引入PET探测器模块, 不仅提升了探测器本身的TOF性能, 还使得PET/MRI 一体机具备可行性. 2011年，西门子公司在全球首先 推出PET/MRI一体机. 近年来, GE公司与联影公司也 分别推出了基于 SiPM、具有TOF功能的PET/MRI产 品. 2019年取得美国食品药品监督管理局(Food and Drug Administration, FDA)上市许可的另一款重量级 核医学影像设备是轴向视野 $2 \mathrm{~m}$ 的PET/CT(图1). 这款 设备由美国U. C. Davis大学研究团队与我国联影公司 合作研制，采用了联影公司的高分辨率PET探测器技 术(https://usa.united-imaging.com). 虽然在基础原理和 系统设计上并没有较大的创新，但是 $2 \mathrm{~m}$ 长轴向视野 与25 30倍灵敏度提升，在工程技术方面的实现难度 以及对图像重建算法的要求都具有巨大挑战, 其对核 医学行业的影响力庸置疑. 临床研究和应用虽然刚 


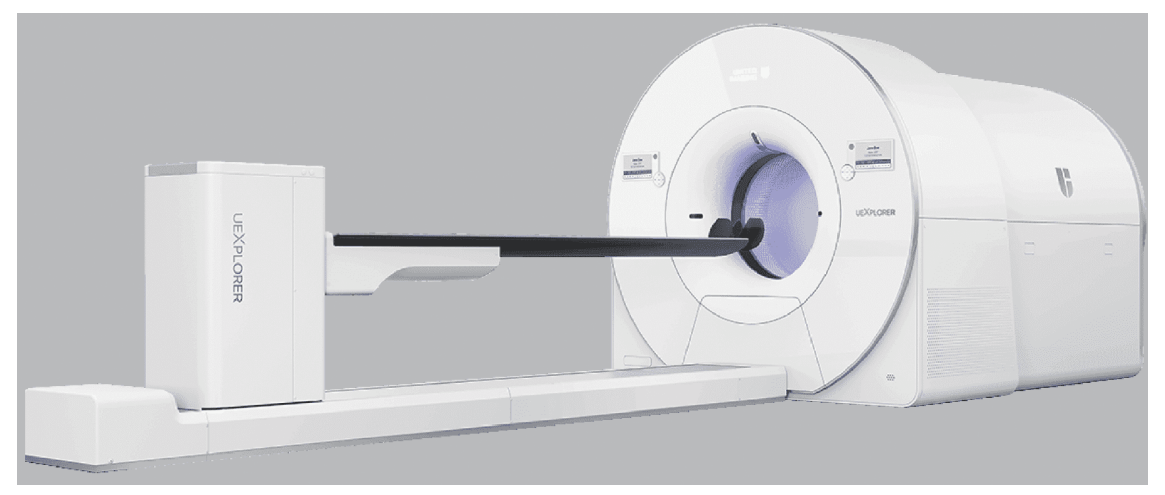

图 1 联影公司 uExplorer $2 \mathrm{~m}$ 长轴向视野PET/CT

Figure 1 uExplorer PET/CT of $2 \mathrm{~m}$ axial field of view by United Imaging Healthcare Co. Ltd

刚展开, 但前景值得期待.

与PET类似, 一种新型的碲锌铬半导体探测器 $(\mathrm{CdZnTe})$ 也为SPECT设备的创新提供了重要支持。碲 锌铬探测器也是由阵列单元组成模块, 每个单元可以 直接将伽马光子与半导体作用产生的电子空穴对转化 为电流输出, 并进一步通过专用集成电路(application specific integrated circuits, ASIC)转化为该伽马光子能 量与位置的数字信号. 与传统基于整块 $\mathrm{NaI}$ 闪炼晶体与 真空光电倍增管阵列的SPECT探测器相比, 碲锌铬探 测器优点在于空间分辨率更高，能量分辨率更高，模 块体积更小，模块之间的死区面积更小，更容易组成 环形或弧形探测器; 缺点在于设备成本高, 并且厚度 因为工艺原因难以增加到 $10 \mathrm{~mm}$ 以上，对高能伽马光 子的探测能力较弱. 目前至少有四款临床SPECT设备 采用了该种探测器, 分别是 GE公司的NM530心脏专用 SPECT、双探头通用型SPECT/CT NM670-CZT以及 Spectrum Dynamics公司的心脏专用DSPECT和环状探 测器通用型SPECT Veriton (https://www.gehealthcare. com). 这四款机型中, 两款心脏专用SPECT在临床上获 得了较为普遍的认可, 两款通用机型在临床的推广并 不理想, 其主要原因在于探测器只是决定SPECT成像 性能的一个环节, 包括准直器、重建算法在内的系统 技术方案才真正决定了SPECT整体技术指标和产品的 成败.上述SPECT新技术的应用情况说明，小动物 SPECT设备中取得成功的小视野聚焦成像技术较好地 拓展到了人体心脏这一中小视野应用领域，但在进一 步应用到全身大视野成像场景时遇到了严峻的挑战, 至今尚未取得突破性的进展.
在放射性药物方面, 针对疾病的精准诊治, 世界各 国都在加强特异性靶向药物的研发 (表 1$)^{[18 \sim 32]}$. ${ }^{68} \mathrm{Ga}-$ DOTA-TOC作为诊断神经内分泌肿瘤的新型放射性显 影剂于 2019年获FDA批准上市, 是FDA批准的第一 个 ${ }^{68} \mathrm{Ga}$ 标记的放射性药物 ${ }^{[24,33]}$. 靶向前列腺特异性膜 抗原(prostate-specific membrane antigen, PMSA) 的 ${ }^{68} \mathrm{Ga}-\mathrm{PSMA}-617$ 显像剂在前列腺癌的诊断中显示出 良好的效果, 目前正在开展III期临床研究 ${ }^{[25]}$. 2019年, 德国多中心研究成果表明, 靶向成纤维细胞激活蛋白 (fibroblast activation protein, FAP) 的 ${ }^{68}$ Ga-FAPI-04显像 剂可以检测 80 例患者的 28 种不同肿瘤原发灶及转移 灶 ${ }^{[26]}$, 这是近年来快速发展的诊断用放射性药物的一 个重大突破.

在神经退行性疾病方面, 2012年Lilly公司的Amy$\operatorname{vid}\left(\left[{ }^{18} \mathrm{~F}\right] \text { Florbetapir }\left({ }^{18} \mathrm{~F}-\mathrm{AV} 45\right) \text { 注射液 }\right)^{[30]}$ 获得FDA批 准上市, 成为首个应用于阿尔茨海默病(Alzheimer disease, AD)诊断用放射性药物; 2013年和2014年, FDA又 陆续批准GE公司的Vizamyl $\left(\left[{ }^{18} \mathrm{~F}\right]\right.$ Flutemetamol $\left({ }^{18} \mathrm{~F}\right.$ PIB) 美他酚注射液 $)^{[31]}$ 和Pirama公司的Neuraceq $\left(\left[{ }^{18} \mathrm{~F}\right]\right.$ Florbetaben氟比他班注射液 ${ }^{[32]}$ 用于 $\mathrm{AD}$ 的诊断，有望 实现对 $\mathrm{AD}$ 的早期诊断和早期干预.

近年来治疗用放射性药物日益受到重视并也有较

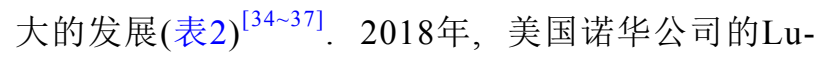
tathera $\left({ }^{177} \mathrm{Lu}\right.$-DOTA-TATE) 获得FDA批准上市, 这是全 球首个获批的多肽受体放射性治疗药物, 用于治疗生 长抑素受体(somatostatin receptor, SSTR)阳性的胃肠 胰腺神经内分泌肿瘤. 该公司还有一款处于III期临床 研究阶段的放射性治疗药物 ${ }^{177}$ Lu-PSMA-617, 用于治 
表 1 近 10 年获批上市以及临床研究阶段的诊断用放射性药物

Table 1 Diagnostic radiopharmaceuticals approved for marketing and clinical research in the last decade

\begin{tabular}{|c|c|c|c|c|c|c|}
\hline 药物名称 & 配体 & 核素 & 靶点 & 研发公司或机构 & 疾病 & $\begin{array}{c}\text { 批准时间或临床试验阶 } \\
\text { 段 }\end{array}$ \\
\hline${ }^{99 \mathrm{~m}}$ Tc-3PRGD2 & 多肽 & ${ }^{99 \mathrm{~m}} \mathrm{Tc}$ & 整合素 $\alpha v \beta 3$ & 北京大学 & $\begin{array}{c}\text { 肺癌及其淋巴结 } \\
\text { 转移 }\end{array}$ & $\begin{array}{c}\text { III期临床试验 } \\
\text { (Chinadrugtrials登记号: } \\
\text { CTR20191465) }\end{array}$ \\
\hline $\begin{array}{c}{ }^{99 \mathrm{~m}} \text { Tc-HYNIC-H10F } \\
{ }^{99 \mathrm{~m}} \text { Tc-HPArk2 }\end{array}$ & 多肽 & ${ }^{99 \mathrm{~m}} \mathrm{Tc}$ & HER2 & 北京大学 & 乳腺癌 & 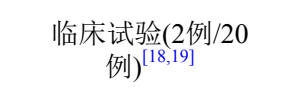 \\
\hline${ }^{99 m}$ Tc-GSA注射液 & 蛋白 & ${ }^{99 \mathrm{~m}} \mathrm{Tc}$ & $\begin{array}{c}\text { 去唾液酸糖蛋白受 } \\
\text { 体 }\end{array}$ & $\begin{array}{c}\text { 容成医学技术无锡 } \\
\text { 有限公司 }\end{array}$ & 肝功能 & $\begin{array}{l}\text { I 期临床试验(China- } \\
\text { drugtrials登记号: } \\
\text { CTR20191555) }\end{array}$ \\
\hline 碘 $\left[{ }^{123} \mathrm{I}\right]$ 氟潘(ioflupane $\left[{ }^{123} \mathrm{I}\right]$ ) & 小分子 & ${ }^{123} \mathrm{I}$ & 多巴胺转运蛋白 & 美国GE医疗集团 & 帕金森综合征 & $\begin{array}{l}\text { 2011年FDA批准上市 } \\
\text { (DaTscan) }^{[20]}\end{array}$ \\
\hline${ }^{99 \mathrm{~m}}$ Tc-FAPI-34 & 小分子 & ${ }^{99 \mathrm{~m}} \mathrm{Tc}$ & $\begin{array}{c}\text { 成纤维细胞激活蛋 } \\
\text { 白(FAP) }\end{array}$ & 德国海德堡大学 & 胰腺癌和卵巢癌 & 临床试验 $(2 \text { 例 })^{[21]}$ \\
\hline${ }^{99 \mathrm{~m}}$ Tc-PSMA-I\&S & 小分子 & ${ }^{99 \mathrm{~m}} \mathrm{Tc}$ & $\begin{array}{c}\text { 前列腺特异性膜抗 } \\
\text { 原(PSMA) }\end{array}$ & $\begin{array}{c}\text { 德国慕尼黑工业 } \\
\text { 大学 }\end{array}$ & $\begin{array}{c}\text { 前列腺癌及淋巴结 } \\
\text { 转移 }\end{array}$ & 临床试验(1例 $)^{[22]}$ \\
\hline $\begin{array}{l}{ }^{99 \mathrm{~m}} \text { Tc-DTPA-mannosyl-dex- } \\
\text { tran }\end{array}$ & 大分子 & ${ }^{99 \mathrm{~m}} \mathrm{Tc}$ & CD206 & $\begin{array}{c}\text { 美国Navidea生物 } \\
\text { 制药公司 }\end{array}$ & $\begin{array}{l}\text { 乳腺癌或黑色素瘤 } \\
\text { 的淋巴结定位 }\end{array}$ & $\begin{array}{l}\text { 2013年FDA批准上市 } \\
\text { (Lymphoseek) }\end{array}$ \\
\hline${ }^{99 \mathrm{~m}} \mathrm{Tc}-\mathrm{NM}-01$ & 单域抗体 & ${ }^{99 \mathrm{~m}} \mathrm{Tc}$ & PD-L1 & $\begin{array}{c}\text { 上海交通大学 } \\
\text { 医学院 }\end{array}$ & 非小细胞肺癌 & 临床试验(16例) ${ }^{[23]}$ \\
\hline 氟 $\left[{ }^{18} \mathrm{~F}\right]$ 阿法肽 & 多肽 & ${ }^{18} \mathrm{~F}$ & 整合素 $\alpha v \beta 3$ & $\begin{array}{c}\text { 江苏原子医学 } \\
\text { 研究所 }\end{array}$ & 肺癌和乳腺癌 & $\begin{array}{l}\text { I 期临床试验(China- } \\
\text { drugtrials登记号: } \\
\text { CTR20192146) }\end{array}$ \\
\hline${ }^{68}$ Ga-DOTA-TOC & 多肽 & ${ }^{68} \mathrm{Ga}$ & $\begin{array}{c}\text { 生长抑素受体 } \\
\text { (SSTR) }\end{array}$ & $\begin{array}{c}\text { 美国UIHC-PET成像 } \\
\text { 中心 }\end{array}$ & 神经内分泌肿瘤 & $\begin{array}{l}\text { 2019年FDA批准上市 } \\
\text { (TOC scan) }{ }^{[24]}\end{array}$ \\
\hline${ }^{68}$ Ga-PSMA-617 & 小分子 & ${ }^{68} \mathrm{Ga}$ & PSMA & 瑞士诺华制药公司 & 前列腺癌 & III期临床试验 ${ }^{[25]}$ \\
\hline${ }^{68}$ Ga-FAPI-04 & 小分子 & ${ }^{68} \mathrm{Ga}$ & FAP & 德国海德堡大学 & $\begin{array}{l}\text { 胰腺导管腺癌、结 } \\
\text { 直肠癌、头颈癌等 }\end{array}$ & $\begin{array}{c}\text { 临床试验(80例, 28种 } \\
\text { 肿瘤) }\end{array}$ \\
\hline${ }^{68}$ Ga-cycratide & 多肽 & ${ }^{68} \mathrm{Ga}$ & 整合素 $\alpha v \beta 6$ & 北京大学 & 胰腺癌 & 临床试验(2例) ${ }^{[27]}$ \\
\hline $\mathrm{Al}^{18} \mathrm{~F}$-PSMA-BCH & 小分子 & ${ }^{18} \mathrm{~F}$ & PSMA & 北京大学肿瘤医院 & 前列腺癌 & 临床试验(11例) $)^{[28]}$ \\
\hline${ }^{64} \mathrm{Cu}$-NOTA-Trastuzumab & 抗体 & ${ }^{64} \mathrm{Cu}$ & HER2 & 北京大学肿瘤医院 & 胃癌及转移灶 & 临床试验(1例) ${ }^{[29]}$ \\
\hline${ }^{18}$ F-Florbetapir & 小分子 & ${ }^{18} \mathrm{~F}$ & $\beta$-淀粉样蛋白 & 美国Lilly公司 & 阿尔茨海默病 & $\begin{array}{c}\text { FDA } 2012 \text { 年批准上市 } \\
\text { (Amyvid) }^{[30]}\end{array}$ \\
\hline${ }^{18}$ F-Flutemetamol & 小分子 & ${ }^{18} \mathrm{~F}$ & $\beta$-淀粉样蛋白 & 美国GE医疗集团 & 阿尔茨海默病 & $\begin{array}{c}\text { FDA } 2013 \text { 年批准上市 } \\
\text { (Vizamyl) }^{[31]}\end{array}$ \\
\hline${ }^{18}$ F-Florbetaben & 小分子 & ${ }^{18} \mathrm{~F}$ & $\beta$-淀粉样蛋白 & Pirama公司 & 阿尔茨海默病 & $\begin{array}{c}\text { FDA 2014年批准上市 } \\
\text { (Neuraceq) }\end{array}$ \\
\hline
\end{tabular}

疗转移性去势抵抗性前列腺癌 ${ }^{[35]}$. 同年, FDA批准了 Progenics Pharmaceuticals的Azedra $\left({ }^{131}\right.$ I-iobenguane) 上 市，是美国第一种被批准用于治疗两种罕见肾上腺肿 瘤(嗜铬细胞瘤或副神经节瘤)的药物(https://www.fda. gov). 拜耳制药公司的Xofigo ${ }^{223} \mathrm{RaCl}_{2}$ 注射剂)于 2013 年获FDA批准上市, 用于治疗有骨转移症状和未知原 因内脏转移的去势抵抗性前列腺癌，这是首个获批上 市的 $\alpha$ 粒子放射性治疗药物 ${ }^{[36]}$.

\section{3 我国核医学的发展及未来}

我国核医学起步于 20 世纪50年代中期，与美国以 及欧洲各国基本同步. 经过几代核医学人的共同努力, 我国核医学事业也有了长足的进步和发展，在科研以 及临床转化方面, 部分研究单位及医院核医学科室达 到了世界先进技术水平．但是在临床普及、产业化和 学科整体实力方面，与核医学发展较好的国家和地区 
表 2 近 10年获批上市以及临床研究阶段的治疗用放射性药物

Table 2 Therapeutic radiopharmaceuticals approved for marketing and clinical research in the past decade

\begin{tabular}{|c|c|c|c|c|c|c|}
\hline 药物名称 & 配体 & 核素 & 靶点 & 研发公司或机构 & 疾病 & $\begin{array}{c}\text { 批准时间或临床试验阶 } \\
\text { 段 } \\
\end{array}$ \\
\hline${ }^{177}$ Lu-DOTA-TATE & 多肽 & ${ }^{177} \mathrm{Lu}$ & SSTR & 瑞士诺华制药公司 & $\begin{array}{l}\text { 胃肠胰神经 } \\
\text { 内分泌肿瘤 }\end{array}$ & $\begin{array}{c}\text { FDA 2018年批准上市 } \\
\text { (Lutathera) }{ }^{[34]}\end{array}$ \\
\hline${ }^{177}$ Lu-PSMA-617 & 小分子 & ${ }^{177} \mathrm{Lu}$ & PSMA & 瑞士诺华制药公司 & 前列腺癌 & III期临床试验 ${ }^{[35]}$ \\
\hline${ }^{131}$ I-iobenguane & 抗体 & ${ }^{131} \mathrm{I}$ & $\begin{array}{c}\text { 去甲肾上腺素 } \\
\text { 转运体 }\end{array}$ & Progenics制药公司 & 罕见肾上腺瘤 & $\begin{array}{c}\text { FDA 2018年批准上市 } \\
\text { (Azedra) }\end{array}$ \\
\hline${ }^{223} \mathrm{RaCl}_{2}$ & None & ${ }^{223} \mathrm{Ra}$ & Calcimimetic & 德国拜耳制药公司 & 前列腺癌有骨转移 & 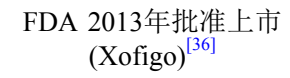 \\
\hline${ }^{177}$ Lu-DOTA-EB-TATE & 多肽 & ${ }^{177} \mathrm{Lu}$ & SSTR & $\begin{array}{c}\text { 北京协和医院与美国 } \\
\mathrm{NIH}\end{array}$ & 神经内分泌肿瘤 & 临床试验(8例 $)^{[37]}$ \\
\hline
\end{tabular}

相比, 还有很大的差距.

临床普及方面，2012年，全世界约有20000台 SPECT，3000台PET，年显像病例数 3000 万，同期我国 只有 600 余台SPECT，不到200台PET，年显像病例数 200 万例. 截至2019年, 我国核医学设备PET、PET/CT 和PET/MRI约为500台, SPECT以及SPECT/CT达到 900 台，年检查病例数超过 260 万. 虽然我国核医学设 备数量在逐年递增, 但与美国的核医学设备保有量相 比仍然差距很大. 美国目前有PET设备约2000台, SPECT 设备约 15000 台, 年检查病例数 2000 万人次左 右. 如果以每百万人拥有核医学设备数量计算, 美 国、日本、韩国以及台湾地区都是我们的十倍或者数 十倍，这与我国经济社会发展和人民群众的需求严重 不匹配.

在设备研制和产业化方面，SPECT设备国产化的 工作可以追溯到 20 世纪 80 年代由中国核工业总公司牵 头、多部委和医院参与的项目 ${ }^{[38]}$. 遗憾的是, 该项目 最终没能成功实现产业化，主要原因有两个，一是当 时国内市场需求、人才团队、产业链配套等方面都不 足以支撑高端医疗器械的产业化, 二是以市场化为目 标的研发思路和实施体系在当时还不够成熟和完善. 直至2005年前后，北京滨松光子技术有限公司才在日 本母公司的技术和资源支持下，完成了首台国产单探 头SPECT的研制和产业化 ${ }^{[39]}$. 但受限于产品自身技术 水平以及滨松公司在行业的综合影响力, 该产品市场 推广也未能取得出色的成绩. 2011年之前，我国核医 学设备基本依赖于进口．从2011年开始，在恶性肿瘤 诊疗市场需求旺盛、社会经济发展水平提高、产业化
配套相对成熟、国家政策引导以及资本市场看好的多 重因素推动下, 核医学影像设备的国产化蓬勃发展. 截 至2019年, 国内已有至少7家内资公司取得了临床PET/ CT、PET/MR产品的上市许可, 2家内资公司有SPECT 产品在市场上推广, 其中联影公司的PET/CT系列产品 在技术水平和市场推广成绩上都达到甚至超越了同类 进口产品的水平.

目前我国临床上常规使用的SPECT药物和PET药 物都已实现国产化, 但都是国外已有的药物, 还没有我 国自主研制的放射性药物获得批准在临床上使用. 据 2018年核医学现状普查结果显示，国内使用SPECT药 物的 644 家医疗机构，自己制备药物为主的不到 $40 \%$, 大部分单位不具备放射性药物 3 类和 4 类许可证，没有 自己制备放射性药物的条件，主要靠药物中心提供药 物. 在PET药物方面，全国有107家单位可以制备F-18 标记药物, 37家单位可以制备C-11标记药物, 22家单 位可以制备N-13标记药物，6家单位可以制备Ga-68标 记药物 ${ }^{[40]}$. I-124、Cu-64和Zr-89等新型正电子核素在 国内也实现了小规模生产用于科研. Tc-99m、I-131和 Sr-89这些临床常规使用的核素还主要依赖于进口.

从进口到国产化替代, 是一个飞跃. 我国核医学经 过60余年的积累, 如何从国产化替代到自主创新, 走符 合中国国情、具有中国特色的核医学分子影像技术与 产品研发路线, 将是我国核医学未来的发展重点. 目前 我国研发的一些PET显像剂也进入临床转化研究阶段, 其中靶向整合素 $\alpha_{\mathrm{v}} \beta_{6}$ 的 ${ }^{68} \mathrm{Ga}-\mathrm{cycratide}$ 的研究结果显示 出对肤腺癌有较好的肿瘤检测能力 ${ }^{[27]}$; $\mathrm{Al}^{18}$ FPSMA$\mathrm{BCH}$ 在前列腺癌检测方面也具有较好的检测能力 ${ }^{[28]}$; 
${ }^{64} \mathrm{Cu}$-NOTA-Trastuzumab在胃癌及其肝转移灶的检测 方面具有较好的应用前景 ${ }^{[29]}$. 在放射性治疗药物方面, 北京协和医院朱朝晖教授与美国国立卫生研究院陈小 元教授课题组合作在 8 例晚期神经内分泌肿瘤 (neuroendocrine tumor, NET) 患者中进行了长效药物 ${ }^{177} \mathrm{Lu}-$ DOTA-EB-TATE和 ${ }^{177}$ Lu-DOTA-TATE的临床试验研 究, 结果显示, ${ }^{177}$ Lu-DOTA-EB-TATE在NET中具有更 高的摄取和更长的滞留时间, 同时NET患者对该药物 具有很好的耐受. 这些研究结果表明, ${ }^{177}$ Lu-DOTAEB-TATE在治疗NET中具有较大潜力 ${ }^{[37]}$. 上述这些药 物目前还仅仅用于临床科研, 不能作为放射性药品推 广应用。

北京大学研发的用于SPECT显像的新型特异性肿 瘤显像剂 ${ }^{99 \mathrm{~m}} \mathrm{Tc}-3 \mathrm{PRGD} 2$, 以及江苏原子医学研究所研 发的用于肿瘤PET显像的 ${ }^{18}$ F-PRGD2 都是自主研发的1 类新药, 都获得了国家药品监督管理局批准的临床试 验批件, 目前分别进入临床III期和 I 期试验阶段, 走 在了世界同类药物研究与产业化的前列. 与PET技术 相比, SPECT核医学分子影像技术的设备及药物成本 更低, 临床普及率更高, 应用基础更好, 因此更适于中 国国情. 目前, 国家药品监督管理局药品审评中心批准 的正在开展临床试验的SPECT显像剂还有: 用于肝病 患者肝细胞功能显像的 ${ }^{99 \mathrm{~m}} \mathrm{Tc}-\mathrm{GSA}$ 注射液(注射用亚锡 半乳糖酰人血清白蛋白二亚乙基三胺五乙酸盐), 用于 嗜铬细胞瘤和副神经节瘤诊断的 ${ }^{131} \mathrm{I}-\mathrm{MIBG}\left(\right.$ 碘 $\left[{ }^{131} \mathrm{I}\right]$ 苄 胍注射液), 用于帕金森综合征患者诊断的碘 $\left[{ }^{123} \mathrm{I}\right]$ 氟潘 (ioflupane $\left[{ }^{123} \mathrm{I}\right]$ ).

目前临床使用的SPECT/CT 是双探头设备, 其分辨 率和灵敏度远远不如PET/CT. 为了充分发挥 ${ }^{99 \mathrm{~m}} \mathrm{Tc}-$ 3PRGD2的临床价值, 为核医学领域带来真正的变革 性技术, 佛山原子医疗设备有限公司在国际上率先提 出人工智能引导的用于人体全身的全环SPECT/CT的 概念 ${ }^{[41]}$, 该设备获得国家自然科学基金委国家重大科 研仪器研制项目的资助. 评审专家认为: “设备的设计 思想突破现有 SPECT/CT的原理, 颠覆双探头临床通 用型SPECT/CT设备的传统技术路线, 主要性能指标 接近或达到主流PET/CT水平, 局部空间分辨率达到 $2 \mathrm{~mm}$.” 该设备除在性能方面优于临床现有双探头 SPECT/CT设备以外, 在设备设计理念上着重强调智 能化技术以解决核医学显像流程复杂和目前我国大部 分基层医院核医学临床技术人员专业基础相对薄弱的
问题，以便更快速地推动核医学在基层医院的普及和 发展. 与PET/CT、PET/MRI相比，该设备最大的优势 在于配合临床适用范围广泛、制备简单、成本低廉的 单光子药物, 能够更好地满足基层医院的综合需求, 发 挥最大的边界效益. 在PET/CT和PET/MRI设备研制方 面, 应注重在现有产品基础上, 加强以硅光电倍增管为 代表的核心关键部件的自主研发和引领性探索. 同时 应努力推动正电子医用加速器的国产化, 显著降低其 配置和运行成本，突破限制PET技术应用推广普及的 瓶颈.

将影像医学和信息科学相结合, 使影像医学朝着 精准化、智能化、个性化、网络化方向迅速发展, 这 也是核医学分子影像发展的必然趋势. 从海量的现有 的医学影像数据中挖掘出更多、更好及更有用的精准 医学信息以构筑集数据共享、处理、融合、分析于一 体的影像融合医疗平台, 其图像数据结果可以辅助医 生更好地进行临床疾病的诊断、治疗与监控. 纵观影 像医学发展历史, 每出现一种新的成像技术, 都会促 进影像医学的跨越式发展. 加强与信息科学合作, 不 断挖掘和提取隐含的成像信息, 定会大大拓展核医学 分子影像的应用范畴.

公众对核医学认知少，核医学普及率低，临床对 核医学需求不够强等因素制约着我国核医学的发展. 但随着精准医学的强化, 核医学分子影像迎来了发展 机会, 但同时也面临着挑战. 影响我国放射性药物发 展的因素包括: 从事放射性药物研究的单位以及相关 研究人员都比较少, 而且人员分布分散, 因此难以承 担重点和重大研究项目, 也难以取得重要成果和突破. 已有的放射性药物厂商研发能力和创新能力不足也 是影响我国放射性药物发展的因素之一. 与放射性药 物相比, 我国在核医学影像设备方面发展迅速, 完成 了从进口到国产替代, 已向创新和技术领先发展, 这 得益于市场化机制, 也得益于国家在高端医疗设备研 制方面的重视和投入. 科技创新和解决临床实际问题, 是我国核医学发展的基础, 特别是我国放射性药物的 发展必须在此基础上寻求突破口, 加强人才培养和储 备, 以开放的心态加强产学研合作, 重视市场机制. 只 有自强发展起来, 满足国家重大需求以及国民经济发 展需要, 才会得到国家的重视和社会的认可, 随后进 入良性循环的发展阶段. 与此同时, 我们坚信新一代 SPECT核医学影像技术的诞生具有颠覆性, 必将为核 
医学领域带来变革性技术. 我们期望我国自主研制的 药物和设备(“中国药+中国环”)为癌症的早篎和精准 诊治提出中国方案，为国家医保节省开支，在国民经
济主战场体现价值, 助力实现健康中国目标, 造福广大 人民群众，也期盼中国核医学能引领国际核医学的 发展。

\section{参考文献}

1 Brownell G L. A history of positron imaging. Physics Research Laboratory, Massachusetts General Hospital, 1999, 1-11

2 Mikołajczak R, Garnuszek P. Radiopharmaceuticals in cardiology. Nucl Med Rev Cent East Eur, 2012, 15: 39-45

3 Podrasky A E, Stark D D, Hattner R S, et al. Radionuclide bone scanning in neuroblastoma: Skeletal metastases and primary tumor localization of ${ }^{99 \mathrm{~m}}$ Tc-MDP. Am J Roentgenol, 1983, 141: 469-472

4 Suapang P, Yimman S. The estimation of renal function with ${ }^{99 \mathrm{~m}}$ Tc-DTPA and ${ }^{99 \mathrm{~m}}$ Tc-MAG3. Int J Appl Biomed Eng, 2014, 7: 40-47

5 Parkey R W, Kulkarni P V, Lewis S E, et al. Effect of coronary blood flow and site of injection on ${ }^{99 \mathrm{~m}}$ Tc-PYP detection of early canine myocardial infarcts. Am J Cardiol, 1980, 45: 464

6 Zhou W, Luan Z S, Tang W G, et al. Diagnosis value of pulmonary embolism by pulmonary perfusion SPECT/CT image fusion (in Chinese). J Taishan Med Coll, 2009, 30: 748-750 [周雯, 栾兆生, 唐为国, 等. ${ }^{99 \mathrm{~m}} \mathrm{Tc}-\mathrm{MAA}$ 肺灌注SPECT/CT同机融合显像诊断肺栓塞的价值. 泰山医学 院学报, 2009, 30: 748-750]

7 Yuan L L, Xu H Q, Kan Y, et al. Application value of nuclear medicine imaging in diagnosis and treatment of hyperparathyroidism (in Chinese). J Clin Exp Med, 2015, 22: 1923-1927 [袁磊磊, 徐海青, 㪏英, 等. 核医学显像在甲状旁腺功能六进症诊治中的应用价值. 临床和实验医学杂 志,2015, 22: 1923-1927]

$8 \mathrm{Xu}$ J M, Zhao J, Peng Y, et al. Diagnostic value of radionuclide bone marrow and bone image for hematopoietic disease (in Chinese). Qianwei J Med, 2001, 18: 260-261 [徐景明, 赵军, 彭勇, 等. 利用放射性核素骨髓和骨骼显像诊断血液病. 前卫医药杂志, 2001, 18: 260-261]

9 Song Z, Yang Q D, Zi X H. The methodology of a.ssessment of cerebrovascular reserve capacity (in Chinese). J Neurosci Mental Health, 2003, 3: 269-272 [宋治, 杨期东, 资晓宏. 脑血管功能储备评估的方法学. 神经疾病与精神卫生, 2003, 3: 269-272]

10 Ido T, Wan C N, Casella V, et al. Labeled 2-deoxy-d-glucose analogs. ${ }^{18}$ F-labeled 2-deoxy-2-fluoro-d-glucose, 2-deoxy-2-fluoro-d-mannose and ${ }^{14}$ C-2-deoxy-2-fluoro-D-glucose. J Label Compd Radiopharm, 1978, 14: 175-183

11 Almuhaideb A, Papathanasiou N, Bomanji J. ${ }^{18}$ F-FDG PET/CT imaging in oncology. Ann Saudi Med, 2011, 31: 3-13

12 Tan Y Y, Tian J H, Zhang J M, et al. The value of ${ }^{18}$ F-FLT PET in the diagnosis and clinical evaluation of lung cancer (in Chinese). Chin J Nucl Med, 2007, 27: 65-67 [谭业渘, 田嘉禾, 张锦明, 等. ${ }^{18}$ F-FLT PET显像诊断肺单发结节及评价细胞增殖的价值. 中华核医学杂志, 2007, 27: 65-67]

13 Zheng X, Zhou N K. Application of ${ }^{18}$ F-FMISO PET in tumor diagnosis and treatment (in Chinese). Med J Chin People's Liber Army, 2006, 31: 496-497 [郑昕, 周乃康. ${ }^{18}$ F-FMISO PET在肿瘤诊疗中的应用. 解放军医学杂志, 2006, 31: 496-497]

14 Zhang J M, Tian J H. Application of carbon-11 radiopharmaceuticals in PET-CT for tumors (in Chinese). Int J Rad Med Nucl Med, 2005, 29: 232-237 [张锦明, 田嘉禾. ${ }^{11} \mathrm{C}$ 标记的放射性药物在PET-CT肿瘤诊断上的应用. 国外医学(放射医学核医学分册), 2005, 29: 232-237]

15 Weissleder R. Molecular imaging: Exploring the next frontier. Radiology, 1999, 212: 609-614

16 Weissleder R. Molecular imaging in cancer. Science, 2006, 312: 1168-1171

17 Vandenberghe S, Mikhaylova E, D’Hoe E, et al. Recent developments in time-of-flight PET. EJNMMI Phys, 2016, 3: 3

$18 \mathrm{Wu}$ Y, Li L, Wang Z, et al. Imaging and monitoring HER2 expression in breast cancer during trastuzumab therapy with a peptide probe ${ }^{99 \mathrm{~m}} \mathrm{Tc}-$ HYNIC-H10F. Eur J Nucl Med Mol Imag, 2020, doi: 10.1007/s00259-020-04754-6

19 Wang R, Zhou Y, Du S, et al. ${ }^{99 \mathrm{~m}}$ Tc-HPArk2 SPECT/CT in the evaluation of HER2-positive breast cancer: A pilot prospective study. J Nucl Med, 2020, 61(supplement 1): 556

20 Broski S M, Hunt C H, Johnson G B, et al. Structural and functional imaging in parkinsonian syndromes. Radiographics, 2014, 34: 1273-1292

21 Lindner T, Altmann A, Kraemer S, et al. Design and development of ${ }^{99 \mathrm{~m}}$ Tc labeled FAPI-tracers for SPECT-imaging and ${ }^{188}$ Re therapy. J Nucl Med, 2020, doi: 10.2967/jnumed.119.239731

22 Robu S, Schottelius M, Eiber M, et al. Preclinical evaluation and first patient application of ${ }^{99 \mathrm{~m}} \mathrm{Tc}-\mathrm{PSMA}$-I\&S for SPECT imaging and radioguided surgery in prostate cancer. $\mathrm{J}$ Nucl Med, 2017, 58: 235-242 
23 Xing Y, Chand G, Liu C, et al. Early phase I study of a ${ }^{99 \mathrm{~m}}$ Tc-labeled anti-programmed death ligand-1 (PD-L1) single-domain antibody in SPECT/CT assessment of PD-L1 expression in non-small cell lung cancer. J Nucl Med, 2019, 60: 1213-1220

24 Lv X L, Zhou W C, Lin K L. Overviews of the New Drugs in 2019 Approved by U.S. FDA (in Chinese). Chin J Pharm, 2020, 51: 1-29 [吕训否, 周伟澄, 林快乐. 2019年美国FDA批准上市的新药简介. 中国医药工业杂志, 2020, 51: 1-29]

25 Morigi J J, Stricker P D, van Leeuwen P J, et al. Prospective comparison of ${ }^{18} \mathrm{~F}$-fluoromethylcholine versus ${ }^{68}$ Ga-PSMA PET/CT in prostate cancer patients who have rising PSA after curative treatment and are being considered for targeted therapy. J Nucl Med, 2015, 56: 1185-1190

26 Kratochwil C, Flechsig P, Lindner T, et al. ${ }^{68}$ Ga-FAPI PET/CT: Tracer uptake in 28 different kinds of cancer. J Nucl Med, 2019, 60: 801-805

27 Feng X, Wang Y, Lu D, et al. Clinical translation of a ${ }^{68}$ Ga-labeled Integrin $\alpha v \beta 6$-targeting cyclic radiotracer for PET imaging of pancreatic cancer. J Nucl Med, 2020, doi: 10.2967/jnumed.119.237347

28 Liu T, Liu C, Xu X, et al. Preclinical evaluation and pilot clinical study of $\mathrm{Al}^{18} \mathrm{~F}-\mathrm{PSMA}-\mathrm{BCH}$ for prostate cancer PET imaging. J Nucl Med, 2019, 60: $1284-1292$

29 Guo X, Zhu H, Zhou N, et al. Noninvasive detection of HER2 expression in gastric cancer by ${ }^{64} \mathrm{Cu}-\mathrm{NOTA}$-trastuzumab in PDX mouse model and in patients. Mol Pharm, 2018, 15: 5174-5182

30 Jiang D J, Gu L Q, Zhang Y. Amyvid, a new agent for the diagnosis of Alzheimer's disease (in Chinese). Chin J New Drugs, 2013, 22: 739-742 [江东杰, 顾利强, 章媛. 诊断阿尔茨海默病新药Amyvid. 中国新药杂志, 2013, 22: 739-742]

31 Zhang J Z. Overview of new drugs approved by the FDA in October 2013 (in Chinese). Shanghai Med Pharm J, 2013, 23: 60 [张建忠. 2013 年10 月FDA批准新药概况. 上海医药, 2013, 23: 60]

32 Tang B D. Florbetaben ${ }^{18}$ F (in Chinese). Chin J Med Chem, 2014, 24: 416 [唐百达. 氟比他班(florbetaben ${ }^{18}$ F). 中国药物化学杂志, 2014, 24: 416]

33 Hennrich U, Benešová M. $\left[{ }^{68}\right.$ Ga]Ga-DOTA-TOC: The first FDA-approved ${ }^{68}$ Ga-radiopharmaceutical for PET imaging. Pharmaceuticals, 2020, 13: 38

34 Wei L J. Reviews of FDA new drug approvals in 2018 (I) (in Chinese). Prog Pharm Sci, 2019, 43: 70 -77 [魏利军. 2018年FDA批准的新药评述 ( I ). 药学进展, 2019, 43: 70-77]

35 Hofman M S, Violet J, Hicks R J, et al. $\left[{ }^{177}\right.$ Lu]-PSMA-617 radionuclide treatment in patients with metastatic castration-resistant prostate cancer (LuPSMA trial): a single-centre, single-arm, phase 2 study. Lancet Oncol, 2018, 19: 825-833

36 Jadvar $\mathrm{H}$, Colletti P M. ${ }^{18} \mathrm{~F}-\mathrm{NaF}^{223} \mathrm{RaCl}_{2}$ theranostics in metastatic prostate cancer: treatment response assessment and prediction of outcome. Br J Radiol, 2018, 91: 20170948

37 Zhang J, Wang H, Jacobson O, et al. Safety, pharmacokinetics, and dosimetry of a long-acting radiolabeled somatostatin analog ${ }^{177}$ Lu-DOTA-EBTATE in patients with advanced metastatic neuroendocrine tumors. J Nucl Med, 2018, 59: 1699-1705

38 Anonymous. The feasibility demonstration meeting for the domestic development of the single-photon emission CT tomography device was held in Beijing (in Chinese). Nucl Electron Detect Technol, 1989, 6: 379-380 [匿名. 单光子发射CT断层扫描装置国产化开发可行性论证会在京举 行. 核电子学与探测技术, 1989, 6: 379-380]

39 Zhang S W, Tian J H, He Y J, et al. The quality control and performance tests of domestic BHP6601 SPECT (in Chinese). Inform Med Equip, 2005, 20: 26-29 [张书文, 田嘉禾, 何义杰, 等. 国产BHP6601型SPECT的性能测试与质量控制. 医疗设备信息, 2005, 20: 26-29]

40 Chinese Society of Nclear Medicine. A brief report on the results of the national survey of nuclear medicine in 2018 (in Chinese). Chin J Nucl Med Mol Imag, 2018, 38: 813-814 [中华医学会核医学分会. 2018年全国核医学现状普查结果简报. 中华核医学与分子影像杂志, 2018, 38: 813-814]

41 Deng X, Chen S, Li X, et al. Development of an adaptive clinical SPECT/CT system with full-ring detectors and multi-pinhole. J Nucl Med, 2020, 61(supplement 1): 317 


\title{
Strengthening the independent innovation and development of nuclear medicine molecular imaging in China
}

\author{
CHEN Si ${ }^{1}$, SHI JiYun ${ }^{2} \&$ WANG Fan ${ }^{2,3}$ \\ 1 Foshan Atomical Medical Equipment Ltd, Foshan 528200, China; \\ 2 Key Laboratory of Protein and Peptide Pharmaceuticals, Institute of Biophysics, Chinese Academy of Sciences, Beijing 100101, China; \\ 3 Medical Isotopes Research Center, Peking University, Beijing 100191, China
}

Nuclear medicine imaging plays an irreplaceable role in the diagnosis, staging, prognosis, therapeutic effect evaluation and guiding individualized treatment of diseases, and has been widely used in clinical practice. The development of nuclear medicine imaging depends on the continuous progress of imaging agents and equipment. Nuclear medicine in China has developed for more than 60 years, and it has also completed the transformation of imaging agents and equipments from imports to domestic substitution. Nowadays, nuclear medicine in China has entered the stage of independent innovation. In this paper, the development and current situation of radiopharmaceuticals and nuclear medicine equipments are described, and the development of nuclear medicine in China is also prospected.

nuclear medicine imaging, radiopharmaceutical, SPECT, PET, diagnosis, therapy

doi: $10.1360 / \mathrm{SSV}-2020-0168$ 